\title{
Characterizing Outcomes and Unmet Needs Among Patients in the United States with Mild-to-Moderate Plaque Psoriasis Using Prescription Topicals
}

\author{
Shaloo Gupta · Steven Garbarini · Tara Nazareth · Ibrahim Khilfeh • \\ Halley Costantino · David Kaplan
}

Received: June 23, 2021 / Accepted: September 25, 2021 / Published online: October 14, 2021

(C) The Author(s) 2021

\section{ABSTRACT}

Introduction: Topical therapies are considered first-line treatment in the management of plaque psoriasis (PSO). However, data on patientreported outcomes for topicals are scarce. We designed a survey to record the treatment experience of patients with mild-to-moderate PSO using prescription topicals.

Methods: An online cross-sectional survey was conducted among adult patients on prescription topicals for mild-to-moderate PSO (body surface area $[\mathrm{BSA}] \leq 10 \%)$ in the US. Data on treatment goals, changes in PSO after current treatment, satisfaction with current treatment (assessed with the Treatment Satisfaction Questionnaire for Medication [TSQM]), and treatment adherence (how often current treatment was taken as instructed) were collected. Descriptive analysis was used to evaluate outcomes.

Results: Of the 175 patients with mild-tomoderate PSO who completed the survey,

S. Gupta $(\bowtie) \cdot$ S. Garbarini · H. Costantino

Kantar Health, Malvern, PA, USA

e-mail: shaloo.gupta@kantar.com

T. Nazareth · I. Khilfeh

Amgen Inc., Thousand Oaks, CA, USA

D. Kaplan

Adult and Pediatric Dermatology, Overland Park, KS, USA
$67.4 \%$ were female, with a median age of 55.0 years and 10.8 years since PSO diagnosis. Patients reported (medians) the use of three topicals since diagnosis, with 5 years on the current prescription topical. The top three treatment goals for current topical treatment were improvements in visible skin, 97.1\%; nonskin related symptoms, $62.9 \%$; and social/emotional well-being, $60.0 \%$. Overall, $43.4 \%$ of patients reported 0\% BSA change and 5.7\% reported $\geq 75 \%$ BSA reduction. Approximately $75.0 \%$ each reported improvement in itch and pain symptoms. Embarrassment/self-consciousness because of skin symptoms persisted in $72.6 \%$ of patients. Median TSQM scores for global satisfaction, convenience, and effectiveness ranged between 58 and 72, indicating partial treatment satisfaction, except for side effects, which was high (median: 100). Approximately half of patients (49.7\%) reported not being highly adherent to treatment.

Conclusion: Contrary to their treatment goals, patients with mild-to-moderate PSO using prescription topicals reported partial effectiveness, incomplete symptom resolution, impacted emotional and social well-being, and suboptimal global satisfaction, effectiveness, adherence, and convenience. Our findings highlight several unmet needs among topical-experienced, systemic-naïve patients with mild-tomoderate PSO using prescription topicals. 


\section{PLAIN LANGUAGE SUMMARY}

Psoriasis is a chronic skin disease caused by inflammation in the body. Raised areas, called plaques, are one of the main symptoms. These plaques may be red, flaky, itchy, and/or painful. Living with psoriasis can negatively impact a person's well-being, especially when it affects visible areas such as the head, face, hands and nails, and/or sensitive areas like the genitals. Topical treatments, such as creams and lotions, are often the first therapy a doctor prescribes for psoriasis. Since these treatments are applied directly on the plaques, they can be messy and inconvenient. We conducted an online survey of adults with mild-to-moderate psoriasis, meaning that less than $10 \%$ of their body had plaques. We wanted to learn how they felt about their disease and their prescription topical treatments. Most people had psoriasis for about 10 years and had been using their current prescription topical for about 5 years. Approximately three of four people reported embarrassment/self-consciousness because of skin symptoms. Although three of four people reported some improvement in itch and pain, almost all people desired better resolution of plaques in visible areas. People also reported that: their current prescription topical did not completely resolve their symptoms, their emotional and social well-being was still suboptimal, they did not always apply their therapy as often as instructed, and they were not completely satisfied with their treatment overall. These results highlight the need for additional treatment options for people with mild-tomoderate psoriasis, particularly options that offer improved symptom control and are more convenient to use.

Keywords: Body surface area; Mild-tomoderate plaque psoriasis; Patient-reported outcomes; Prescription topicals; Treatment adherence; Treatment satisfaction

\section{Key Summary Points}

Plaque psoriasis can be classified into mild, moderate, or severe depending on the body surface area affected

Topical treatments are considered as the first-line treatment for plaque psoriasis. However, topicals can be time-consuming to apply, may cause side effects including burning and skin irritation, and do not affect underlying systemic inflammatory pathways to provide lasting resolution of symptoms

Recently published studies assessed the efficacy, safety, and patient preferences for medications in the treatment of moderate-to-severe plaque psoriasis, whereas data on patient-related needs and goals to initiate individualized treatment for improved management of patients with mild-to-moderate plaque psoriasis are limited

This cross-sectional survey was conducted to seek patients' experience on the use of prescribed topical treatment for mild-tomoderate plaque psoriasis who have not received systemic treatment and have limited skin involvement

Despite receiving (medians) three topicals since the diagnosis and the current topical treatment for 5 years, patients with mildto-moderate plaque psoriasis reported partial effectiveness and symptom resolution, impacted emotional and social well-being, suboptimal global satisfaction, effectiveness, adherence, and convenience

Overall study findings demonstrate the need for improved management and therapeutic options for patients with mild-to-moderate plaque psoriasis that help provide better effectiveness and symptom resolution as well as greater patient satisfaction while incurring less burden on the patients 


\section{INTRODUCTION}

Psoriasis is a complex chronic inflammatory autoimmune skin disease that affects around $3.2 \%$ of the population in the US $[1,2]$. In addition to limiting daily activities, occupational function, and sexual function, psoriasis has a negative impact on patients' emotional, social and physical well-being as well as a high impact on psychological well-being [3-7].

Plaque psoriasis (PSO), the most common phenotype of psoriasis, accounts for $>80 \%$ of psoriasis cases [8]. The severity of PSO can be classified based on the body surface area (BSA) affected into mild $(<3 \%)$, moderate $(3-10 \%)$, or severe $(>10 \%)$ [9]. Alternatively, even with BSA $<10 \%$, PSO on exposed skin and sensitive special areas (e.g., genitals, scalp, nails, and palmoplantar) has a great impact on patients' physical and emotional well-being $[9,10]$.

The joint American Academy of Dermatology and National Psoriasis Foundation treatment and management guidelines recommend topical therapies for PSO not involving intertriginous areas [11]. However, topical treatments can be time-consuming and inconvenient to use, and patients may experience side effects, including stinging, burning, pruritis, irritation, and edema [8, 11-13]. Furthermore, topical treatments provide relief from the localized symptoms but do not affect underlying systemic inflammatory pathways [14]. There is limited evidence regarding the impact of topical treatments on the nature of the disease in terms of providing lasting improvement or consistent reduction of symptoms [13].

The management and diagnosis of psoriasis on intertriginous areas (e.g., areas where skin folds); difficult-to-treat, sensitive or hard-toreach areas (e.g., nails, breasts/genitals, or middle of the back); or other highly visible areas (e.g., face, hands, scalp, and neck) may be especially challenging [10]. Owing to the location affected, physicians may not completely evaluate the overall burden of the disease and patients can be reluctant to discuss symptoms [10]. Moreover, actual impact of psoriasis on the quality of life (QoL) may be difficult to evaluate because of limitations associated with existing topical treatment.

Understanding patients' needs and the impact of psoriasis on the physical, psychological, and social well-being may help to facilitate improved management, thereby alleviating disease morbidity, enhancing patients' QoL, and enabling better clinical outcomes [15]. Recently published data have mainly focused on reporting safety, efficacy, and patient preferences for moderate-to-severe PSO $[8,11,16-18]$; however, treatment goals, challenges, and needs in patients with mild-tomoderate PSO remain unaddressed.

The primary objective of this study was to seek patient insights on the perceived effects and satisfaction with the use of prescribed topical therapy for treating mild-to-moderate PSO in topical-experienced, systemic-naïve patients.

\section{METHODS}

\section{Study Design}

This cross-sectional survey was conducted in patients meeting the following eligibility criteria: (1) at least 18 years of age; (2) diagnosed with PSO by a healthcare provider (HCP) at least 6 months before the survey; (3) currently receiving a prescription topical for at least 3 months before the survey; (4) received at least one prescription topical therapy before the survey; (5) self-identified PSO severity as mild or moderate before the start of their current therapy, with no more than ten palm-sized areas of plaques/lesions (i.e., BSA $\leq 10 \%$ ); (6) having no more than four joints affected if co-diagnosed with psoriatic arthritis (PsA); (7) were willing to provide an informed consent statement for the study; and (8) were located in the US. Patients were excluded if they disclosed prior PSO treatment with a systemic agent (i.e., apremilast, biologics, and disease-modifying antirheumatic drugs) or were pregnant/actively trying to get pregnant at the time of the recruitment.

A sequenced approach was used to develop the survey: 
- Patients' elicitation and feedback: $(N=29)$ patients participated in 45-min exploratory qualitative telephone interviews. Outcomes from this phase helped to define concepts that were important for patients with PSO to draft the online survey.

- Pilot testing the survey: additional 45-min telephonic interviews $(N=5)$ were conducted that evaluated the survey and provided extra time for probing and understanding the rationale for responses to ensure that the survey was clear and concise.

- A 45-min online patient survey was fielded $(N=175)$. Eligible patients were provided with a unique de-identified link to complete the survey electronically using either a computer or a tablet. The survey was fielded in the first half of 2020, at the start of the coronavirus disease (COVID-19) pandemic.

\section{Measures}

\section{Patient Demographic, Health, and Insurance Characteristics}

Sociodemographic variables including age, sex, race/ethnicity, marital status, education, household income, and employment status were collected.

Health characteristics included body mass index categories and comorbidities of interest such as acne, atopic dermatitis (eczema), Behçet's disease, cancer (not skin), cerebrovascular disease, depression/anxiety, diabetes, fatigue, gout, high blood pressure or hypertension, high cholesterol (dyslipidemia), inflammatory bowel disease (including Crohn's disease and ulcerative colitis), cardiovascular/cerebrovascular disease, liver abnormalities or liver disease, obesity, osteoarthritis, psoriasis, PsA, rheumatoid arthritis, skin cancer, other, or none.

The data on insurance type, insurance coverage of PSO prescriptions, HCPs prescribing current psoriasis treatment, and number of psoriasis-related visits to $\mathrm{HCP}(\mathrm{s})$ for the past 6 months were collected.

\section{PSO-Relevant Clinical Characteristics: Diagnosis}

The data on chronic PSO characteristics, including the number of years since diagnosis, number of years diagnosed with PsA, and location and number of joints affected by PsA, were collected.

\section{Treatment Characteristics and Treatment Goals}

The data on PSO treatment, including type and duration of current treatment(s), previous treatments, number of different topical treatments used since diagnosis, duration of topical treatment(s), treatment used immediately prior to current treatment, and the type of treatment (e.g., laser, prescription topicals, light/ultraviolet, and over-the-counter/non-prescribed topical treatments), were collected.

Patient treatment goals included psoriasis goals at the start of current treatment and at the start of the previous treatment. The main categories were improvement of visible skin symptoms, improvement of other non-skin-related (non-visible) symptoms, improvement of social/emotional well-being, improvement of adverse health effects, decreased treatment burden, and other (specify). Improvement of visible skin symptoms referred to the clearance of most bothersome skin symptoms, clearance of most bothersome body areas, and total or near skin clearance. Improvement of other nonskin-related (non-visible) symptoms referred to improved sleep or less itching, burning, or bodily pain. Improvement of social/emotional well-being referred to normal working/school/ home life, better social interactions, improved self-esteem, decreased level of embarrassment/self-consciousness, and feeling happier. Decrease in treatment burden referred to reduced lifestyle compromises and burden of treatment. Other goals did not apply to any of the previous categories. These categories were stack ranked, with rank 1 being the most important goal. The number of patients ranking each goal as one of their top three most important goals was calculated for both current and prior treatment responses. 


\section{PSO Changes with Current Treatment}

Patients reported the number of palm-sized psoriasis plaques/lesions (current and immediately prior to current treatment) and the severity of psoriasis (scale of 1-10: 1 = very mild to $10=$ very severe [current and immediately prior to current treatment]). The percentage of change in palm-sized psoriasis plaques/lesions with 0 palms was denoted as 0\% BSA [19]. The severity level from the current treatment was also categorized into three options: mild (1-3), moderate (4-7), and severe (8-10).

The reduction in BSA was calculated by subtracting the current number of palm-sized psoriasis plaques/lesions from the immediately prior to current treatment number of palmsized psoriasis plaques/lesions. The result of the reduction in BSA was used to calculate the proportion of patients with $0-25 \%, 25-50 \%$, $50-75 \%$, and $\geq 75 \%$ reduction in BSA. The reduction in BSA was also categorized into three options, $>0$ (improved), $=0$ (no change), and $<0$ (worsened), based on the magnitude and direction of BSA change on the previous versus the current prescription topical treatment. The proportion with clear or almost clear BSA after the treatment was calculated as the proportion with current number of palm-sized psoriasis plaques/lesions equal to 0 or 1 .

Patients provided their responses on a Likert scale $(1=$ extremely to $4=$ not at all $)$ for the current treatment and immediately prior to the start of current topical treatment(s) regarding how itchy, sore, painful, or stinging the skin was and social embarrassment or self-consciousness because of the skin symptoms. Current level of how itchy, sore, painful, or stinging the skin was and current level of how embarrassed or self-conscious because of the skin were combined into the following categories: not at all (4) versus any $(1,2$, or 3$)$. Separately, patients also reported the improvement in pain and itch symptoms ( $1=$ symptom worsened to 4 = symptom completely resolved) from any body area (e.g., scalp, face, and neck) after starting the current treatment(s). Changes in pain and itch symptom categories were combined into two categories: did not improve ( 1 = symptom worsened, $2=$ no change in symptom $)$ and improved (3 = symptom improved, 4 = symptom completely resolved).

Patients also reported body locations affected currently and immediately before the start of the current treatment. Specific body areas were combined into categories. The category of "visible areas" combined face, neck, palms, back of hands, and nails; "special areas" combined scalp, palms, genitals, anal area, areas on the body that flex, soles of feet, and nails; "palmoplantar areas" combined palms and soles of feet; "intertriginous areas" combined genitals, anal area, and areas on the body that flex; "face/neck" combined face and neck; "arms" combined elbows and arms/forearms; "trunk" combined back and chest/abdomen; and "anogenital" combined genitals and anal area. If patients reported any of the body locations in the category affected, then the body area category was considered to be affected. The proportion with symptom resolution on each area was calculated as the patients who had symptoms affecting the body area before the current treatment(s) but did not have symptoms affecting the body area after starting the current treatment(s).

\section{Current Treatment Satisfaction and Adherence}

Current PSO treatment satisfaction was assessed using the Treatment Satisfaction Questionnaire for Medication (TSQM Version 2) [20, 21]. It is an abbreviated 11-item scale derived from the TSQM version 1.4, which comprises four domains: global satisfaction, medication effectiveness, convenience, and side effects. The scores range from 0 to 100 , with higher scores indicating greater satisfaction and lower scores reflecting room for improvement.

Treatment adherence was assessed to provide information on how often current treatment(s) was taken on schedule as instructed $(1=$ nearly all of the time [80-100\%] to $5=$ never/rarely $[<20 \%]$ ) by the product insert. A response of "nearly all of the time (80-100\%)" was considered to be highly adherent, whereas responses of "usually (60-79\%)" were considered as usually adherent; "sometimes (40-59\%)," "once in a while (20-39\%)," and "never/rarely $(<20 \%)$ " responses were 
considered to be not adherent. Furthermore, patients were asked to provide reasons (e.g., side effects, serious adverse effects, impact on daily activities, requiring lifestyle change, burden of treatment handling, and administration) for not taking medication as instructed.

\section{Statistical Analysis}

All data management and analyses were performed using SAS 9.4. Descriptive statistics were used for all study variables, including frequencies and percentages for categorical variables and medians and ranges (minimum [min] to maximum $[\max ])$ for continuous variables to account for the full range of data and any skewness. No statistical testing was performed.

\section{Compliance with Ethics Guidelines}

All study materials (including protocol, qualitative study materials, final questionnaire, and informed consent) were approved by Pearl Institutional Review Board (IRB) before patient engagement (www.pearlirb.com) (protocol no. 18-KANT-180). The study was performed in accordance with the Declaration of Helsinki 1964 and its later amendments.

Patients were recruited by Profiles (a Kantar company) and other panel/recruiting partners such as Global Perspectives. The online panel of Profiles and its partner panels is an opt-in panel, where participants make a conscious decision to participate regularly in surveys. The consumer panel recruits its panel members through opt-in e-mails, co-registration with panel partners, e-newsletter campaigns, banner placements, and affiliate networks. All panelists explicitly agree to be a panel member, register with the panel through a unique e-mail address, and complete an in-depth demographic registration profile. Global Perspectives expert recruiters are patient recruiters, panel companies, moderators, or translators who are trained to validate respondents and guide them through the requirements of the study. All details of the patients are kept in the strictest confidence and are used purely for research purposes only. Neither the information that could personally identify the respondent nor the personal information sought from the patients or about the patients will be released without their prior knowledge and consent.

All patients provided their consent electronically before completing the survey. Patients who completed the survey received incentives, typically reward points or currency offered by their panels or recruiting experts, which are of fair market value for their time to participate in the survey. Patients were able to redeem their points for gift cards/items of their choice through Profiles' online portal and cash incentives between $\$ 5$ and \$50 USD via Global Perspective.

\section{RESULTS}

\section{Patient Demographic, Health, and Insurance Characteristics}

Of the total 175 patients with mild-to-moderate PSO surveyed $(<3 \%$ BSA $[n=85] ; 3-10 \%$ BSA $[n=90])$, the median age was 55.0 years and $67.4 \%$ were female, with most being Caucasian (89.1\%) (Table 1).

Among all the patients, $61.1 \%$ had completed a university/college degree, and an annual income of more than $\$ 100 \mathrm{~K}$ was reported by $26.9 \%$ of the patients. A similar proportion of patients (34.3\% each) reported an income of $<\$ 50 \mathrm{~K}$ and $\$ 50$ to $<\$ 100 \mathrm{~K}$.

About two-thirds $(65.1 \%)$ of the patients with mild-to-moderate PSO were overweight or obese. The median number of additional comorbidities reported were two; commonly reported comorbidities included high cholesterol $(35.4 \%)$, followed by depression/anxiety $(32.0 \%)$, high blood pressure or hypertension (28.6\%), and diabetes (14.9\%).

In total, 159 (90.9\%) patients were insured; of these, $28.0 \%$ had complete coverage and $57.7 \%$ had partial coverage of their current PSO treatment (Table 1). The majority of patients $(73.7 \%)$ were consulting a dermatologist, whereas some patients were consulting another type of clinician (26.3\%), including general or family practitioner $(17.7 \%)$, a nurse practitioner or physician (4.0\%), and an allergist (1.7\%). 
Table 1 Demographic, health, and insurance characteristics of patients with plaque psoriasis (PSO)

Mild-to-moderate PSO

(BSA 0-10\%) $N=175$

\section{Demographic characteristics}

Age (years)

$$
\text { Median (min-max) }
$$$$
55.00(18.00-80.00)
$$

Sex, $n(\%)$

$$
\text { Female }
$$

Location description, $n$ (\%)

$$
\begin{aligned}
& \text { Urban area } \\
& \quad \text { (population }>100,000) \\
& \text { Suburb of a large city } \\
& \text { (population > 100,000) } \\
& \text { Small city (population } \\
& \text { 30,000-100,000) } \\
& \text { Rural or small town } \\
& \quad \text { (population < 30,000) } \\
& \text { Geographic Location, } n(\%)^{a}
\end{aligned}
$$

West

South

Midwest

Northeast

$36(23.4)$

Racelethnicity, n (\%)

$\begin{array}{ll}\text { Hispanic } & 5(2.9) \\ \text { Caucasian } & 156(89.1) \\ \text { African descent } & 2(1.1) \\ \text { Other } & 12(6.9) \\ \text { Completed university education, } n & 107(61.1)\end{array}$

$$
\text { (\%) }
$$

Annual household income, $n$ (\%)

$$
\begin{array}{ll}
<\$ 50 \mathrm{~K} & 60(34.3) \\
\$ 50-<\$ 100 \mathrm{~K} & 60(34.3) \\
>\$ 100 \mathrm{~K} & 47(26.9) \\
\text { Decline to answer } & 8(4.6)
\end{array}
$$

Table 1 continued

Mild-to-moderate PSO

(BSA 0-10\%) $N=175$

BMI category, $n$ (\%)

$\begin{array}{ll}\text { Underweight } & 6(3.4) \\ \text { Normal } & 49(28.0) \\ \text { Overweight } & 54(30.9) \\ \text { Obese } & 60(34.3) \\ \text { Decline to provide weight } & 6(3.4)\end{array}$

\section{Health characteristics}

Number of additional comorbidities per participant

Median (min-max)

$2.00(0.00-9.00)$

Comorbidity, $n$ (\%)

Acne

$20(11.4)$

Atopic dermatitis (eczema)

28 (16.0)

Behcet's disease

$0(0)$

Cancer (not skin)

$12(6.9)$

Depression/anxiety

$56(32.0)$

Diabetes

26 (14.9)

Gout

$5(2.9)$

High BP or hypertension

50 (28.6)

High cholesterol

$62(35.4)$

Inflammatory bowel disease

4 (2.3)

Heart (or cardiovascular) disease

$9(5.1)$

Liver abnormalities or liver

4 (2.3)

disease

Osteoarthritis

$25(14.3)$

Psoriatic arthritis

$4(2.3)$

Rheumatoid arthritis

$4(2.3)$

Skin cancer

7 (4.0)

Other

$10(5.7)$ 
Table 1 continued

\begin{tabular}{ll}
\hline & $\begin{array}{l}\text { Mild-to-moderate PSO } \\
\text { (BSA 0-10\%) N = 175 }\end{array}$ \\
\hline $\begin{array}{l}\text { Insurance information } \\
\text { Health insurance, } n \text { (\%) }\end{array}$ & $159(90.9)$ \\
Private-company/employer & $68(38.9)$ \\
sponsored & \\
Private-self purchased & $26(14.9)$ \\
Medicaid & $24(13.7)$ \\
Medicare & $48(27.4)$ \\
Public-government & $10(5.7)$ \\
employee/past employee & \\
I have other insurance & \\
I have insurance, but I do not & $4(2.3)$ \\
know/am not sure what type & \\
Must 6 months & \\
I do not currently have health & $16(9.1)$ \\
insurance &
\end{tabular}

$B M I$ body mass index, $B P$ blood pressure, $B S A$ body surface area, PSO plaque psoriasis

${ }^{\text {a }} N=154$ total; 21 were missing as they declined to answer or are no longer with the panel
Patients reported a median of one psoriasis-related visit to an HCP over the past 6 months.

Psoriatic arthritis was reported in four patients (2.3\%), whereas the median time since diagnosis was approximately 2 years, with a median of four PsA-affected joints.

\section{PSO-Relevant Clinical Characteristics: Diagnosis}

Patients with mild-to-moderate PSO received their diagnosis at median 10.8 years prior from an HCP (Table 2).

\section{Treatment Characteristics and Treatment Goals}

Patients with mild-to-moderate PSO reported having used a median of three topical PSO treatments since PSO diagnosis (Table 2). Patients reported that they were on the current prescription topical for a median of 5 years while the prior prescription topical treatment was used for a median of approximately 2 years. More than half $(51.4 \%)$ of the patients reported using a concurrent non-prescription topical treatment. Before the current treatment, 59.4\% of the patients reported the use of a non-prescription topical treatment.

Laser treatment $(4.0 \%)$ and light therapy or phototherapy (13.7\%) were used as adjuvants to current treatments in smaller subsets of patients with mild-to-moderate PSO. In addition, patients reported using laser treatment (6.3\%) and light therapy or phototherapy (22.3\%) before starting their current topical treatments.

The top three psoriasis treatment goals as indicated by the patients on current prescription topical treatment included improvement of visible skin symptoms (97.1\%), improvement of non-skin-related symptoms such as improved sleep or less pain $(62.9 \%)$, and improvement of social/emotional well-being (60.0\%) (Fig. 1). Treatment goals remained consistent for both current and prior treatments. 
Table 2 PSO treatment characteristics

\begin{tabular}{|c|c|}
\hline & $\begin{array}{l}\text { Mild-to-moderate PSO } \\
\text { (BSA 0-10\%) } \\
N=175\end{array}$ \\
\hline \multicolumn{2}{|l|}{$\begin{array}{l}\text { Time since chronic PSO diagnosis } \\
\text { (years) }\end{array}$} \\
\hline Median $(\min -\max )$ & $10.75(0.50-70.17)$ \\
\hline \multicolumn{2}{|l|}{$\begin{array}{l}\text { Number of topical treatments } \\
\text { used since diagnosis }\end{array}$} \\
\hline Median $(\min -\max )$ & $3.00(1.00-12.00)$ \\
\hline \multicolumn{2}{|l|}{$\begin{array}{l}\text { Time on current prescription } \\
\text { topical treatment (years) }\end{array}$} \\
\hline $\operatorname{Median}(\min -\max )$ & $5.00(0.25-20.92)$ \\
\hline \multicolumn{2}{|l|}{$\begin{array}{l}\text { Time on prior prescription topical } \\
\text { treatment (years) }\end{array}$} \\
\hline Median $(\min -\max )$ & $2.17(0.08-20.92)$ \\
\hline \multicolumn{2}{|l|}{ Adjuvant treatments, $n$ (\%) } \\
\hline Current laser treatment & $7(4.0)$ \\
\hline Past laser treatment & $11(6.3)$ \\
\hline $\begin{array}{l}\text { Current light therapy or } \\
\text { phototherapy }\end{array}$ & $24(13.7)$ \\
\hline $\begin{array}{l}\text { Past light therapy or } \\
\text { phototherapy }\end{array}$ & $39(22.3)$ \\
\hline $\begin{array}{l}\text { Current non-prescription } \\
\text { topical treatment }\end{array}$ & $90(51.4)$ \\
\hline $\begin{array}{l}\text { Past non-prescription topical } \\
\text { treatment }\end{array}$ & $104(59.4)$ \\
\hline $\begin{array}{l}\text { Other current psoriasis } \\
\text { medication }\end{array}$ & $3(1.7)$ \\
\hline Other past psoriasis medication & $7(4.0)$ \\
\hline
\end{tabular}

$B S A$ body surface area, $P S O$ plaque psoriasis

\section{PSO Changes with Current Treatment}

Patients with mild-to-moderate PSO reported a median BSA reduction of 0 on their current treatment. By BSA category, $45.7 \%$ of patients reported $0-25 \%$ reduction, $24.0 \%$ reported $50 \%$ to $<75 \%$ reduction, and $11.4 \%$ reported 25 to $<50 \%$ reduction; only $5.7 \%$ of patients reported $\geq 75 \%$ reduction in BSA. Overall, $38.3 \%$ of the patients reported clear or almost clear BSA after their current treatment and 29.1\% reported clear or almost clear before starting their current treatment. Patients reported a median severity of 7 (on a scale of 1-10) before starting the current treatment, suggesting they perceived their PSO to be of "moderate" severity. Only $29.1 \%$ of the patients with mild-to-moderate PSO reported their severity as "mild" while taking the current topical (Table 3).

Most patients (96.0\%) reported itchiness, soreness, pain, or stinging in their skin before starting their current topical; $86.9 \%$ of the patients had these symptoms persist on the current topical treatment. In terms of itch and pain improvement specifically, improvement in itch symptoms $(n=160)$ was reported by $74.4 \%$ of the patients and improvement in PSO pain symptoms $(n=76)$ was reported by $73.7 \%$ of the patients, indicating that $>25 \%$ of the patients did not realize improvements. Similarly, before starting their current topical, $85.1 \%$ of the patients reported being embarrassed or self-conscious because of their skin; $72.6 \%$ of the patients reported this feeling while on current treatment.

Persistent PSO in one or more special body areas was reported by $93.0 \%$ of the patients. Few patients $(3.8 \% ; n=26)$ reported PSO resolution on nails. Resolution of PSO on other special body areas, such as scalp (8.8\%), intertriginous $(20.9 \%)$, palmoplantar $(28.1 \%)$, and any of the aforementioned areas (7.0\%), was reported (Table 3). The proportion of mild-to-moderate patients experiencing symptoms on different body areas with current treatments is depicted in Fig. 2. Similar proportions of patients continued to experience symptoms on the difficultto-treat areas (75.4\%), scalp (53.1\%), and intertriginous areas $(30.3 \%)$ from the time of their previous treatment while on their current treatment, highlighting the lack of improvement. 


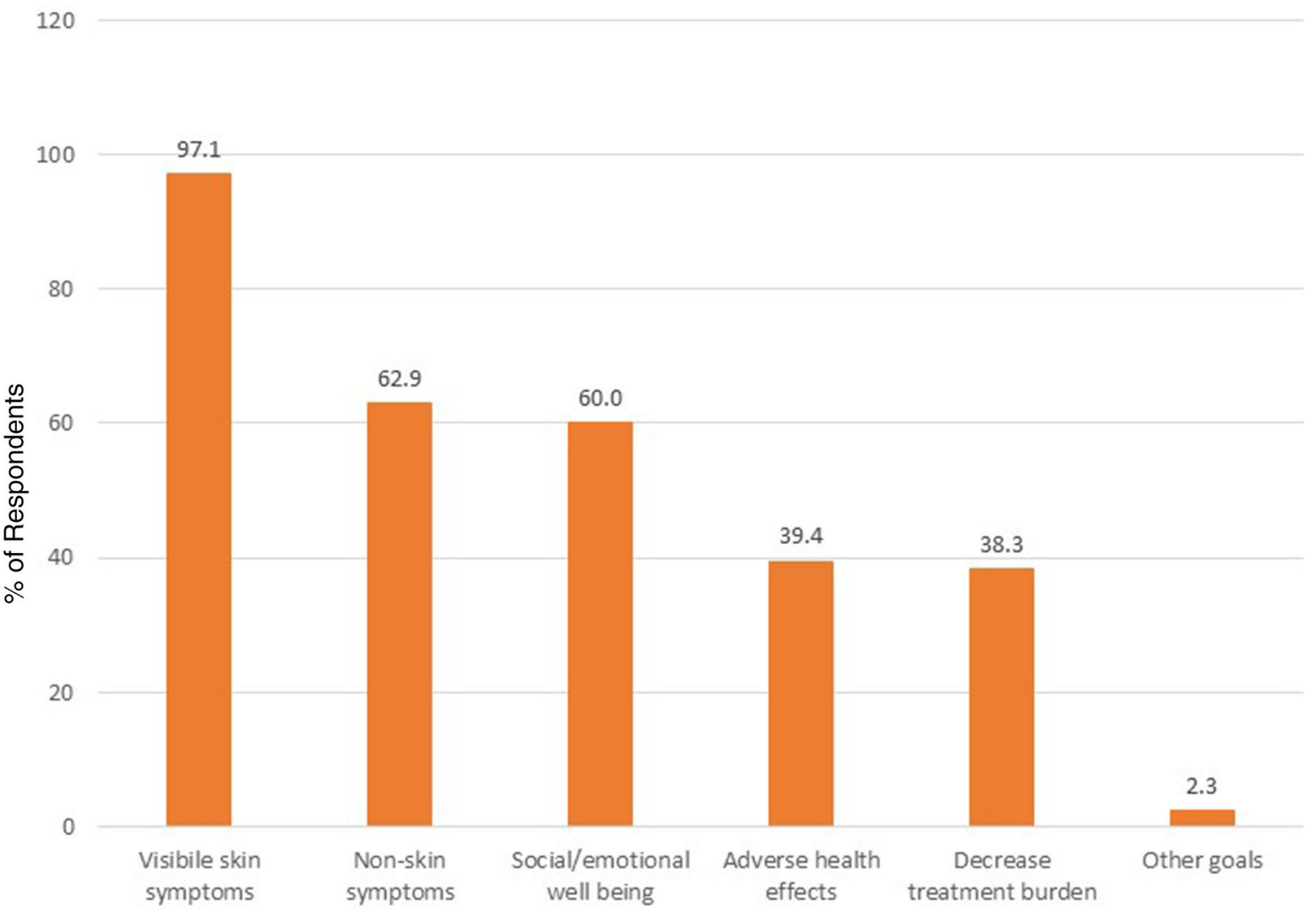

Fig. 1 Top three treatment goals indicated by patients with mild-to-moderate PSO and on current prescription topical. PSO plaque psoriasis. Visible skin symptoms refer to clearance of bothersome skin symptoms and body areas. Other non-skin symptoms refer to improved sleep or less itching, burning, or bodily pain. Social/emotional well-

\section{Current Treatment Satisfaction}

Median TSQM scores were reported for global satisfaction (66.7), effectiveness (58.3), side effects (100.0), and convenience (72.2) (Fig. 3).

\section{Current Treatment Adherence}

Almost half (49.7\%) of the patients with mildto-moderate PSO reported not being highly adherent; only $28.6 \%$ of the patients reported being usually adherent to their current topical medication (i.e., together defined as taking treatment almost always or usually as directed). Overall, $21.1 \%$ of the patients reported adherence as sometimes (13.7\%) or once in while $(4.6 \%)$ or never/rarely (2.9\%) (Fig. 4$)$. The most being refers to normal working/school/home life, better social interactions, improved self-esteem, and feeling happier. Decrease in treatment burden encompasses reduced lifestyle compromises and burden of treatment. Other consists of goals that do not apply to any of the previous categories

frequently reported reasons for nonadherence included forgetfulness (22.9\%), frequency of application $(16.6 \%)$, consistency of the treatment (12.6\%), difficulty in application (10.9\%), inadequate symptom control, and duration of application (9.1\% each) (Fig. 5).

\section{DISCUSSION}

This cross-sectional, online survey evaluated patient-reported outcomes in terms of perceived treatment effectiveness and satisfaction with the use of prescribed topical treatment for mildto-moderate PSO. The study included patients who were diagnosed with PSO for about 10.8 years and on current topical medication for 5.0 years, after using topical treatments 
Table 3 PSO changes with current treatment

Mild-to-moderate
PSO (BSA 0-10\%)
$N=175$

\section{BSA (No. of Palms)}

Current number of palms of psoriasis plaques/lesions

Median (min-max)

$2.00(0.00-12.00)$

Prior number of palms of psoriasis plaques/lesions

Median (min-max)

$3.00(0.00-10.00)$

Reduction in BSA

Median (min-max) $0.00(-7.00$ to 9.00$)$

Proportion of patients reporting reduction in BSA, $n$ (\%)

$<0 \%$ reduction

$23(13.1)$

$0-<25 \%$ reduction

$80(45.7)$

$25-<50 \%$ reduction

$20(11.4)$

$50-<75 \%$ reduction

$42(24.0)$

$\geq 75 \%$ reduction

$10(5.7)$

Proportion with clear or almost clear BSA (0-1) after treatment, $n(\%)$

Not clear $108(61.7)$

Clear/almost clear

$67(38.3)$

Reduction in number of palms of psoriasis plaques/lesions (category), $n$ (\%)

$>0$ [improved]

$=0[$ no change $]$

$<0$ [worsened]

\section{Psoriasis severity}

Severity after starting current treatment, $n$ (\%)

Mild

Moderate

$70(40.0)$

Severe

$54(30.9)$

Current psoriasis severity level (scale of 0 'very mild' to 10 'very severe')

Median $(\min -\max )$

$5.00(1.00-10.00)$
Table 3 continued

\section{Mild-to-moderate \\ PSO (BSA \\ $0-10 \%) N=175$}

Prior psoriasis severity level (scale of 0 'very mild' to 10 'very severe')

Median (min-max)

$7.00(1.00-10.00)$

Level of itchiness, soreness, pain or stinging

Current level of itchiness, soreness, pain, or stinging from skin, $n(\%)$

Any

$152(86.9)$

Not at all

$23(13.1)$

Prior level of itchiness, soreness, pain, or stinging from skin, $n$ (\%)

Any

$168(96.0)$

Not at all

$7(4.0)$

\section{Level of embarrassment / self-consciousness}

Current Level of embarrassment or self-consciousness because of skin, $n(\%)$

Any $127(72.6)$

Not at all $48(27.4)$

Prior Level of embarrassment or self-consciousness because of skin, $n(\%)$

Any

Not at all

$26(14.9)$

\section{Symptom improvement}

Proportion with improved itch symptoms $[N=160], n(\%)$

Did not improve

$41(25.6)$

Improved

$119(74.4)$

Missing

15

Proportion with improved pain symptoms $[N=76], n$ (\%)

Did not improve

$20(26.3)$

Improved

$56(73.7)$

Missing

99 
Table 3 continued

Mild-to-moderate

PSO (BSA

$0-10 \%) N=175$

\section{Area resolution}

Proportion experiencing symptom resolution on special areas ${ }^{a}$ $[N=142], n(\%)$

No

$132(93.0)$

Yes

$10(7.0)$

Not effected

33

Proportion experiencing symptom resolution on scalp $[N=102], n(\%)$

No

$93(91.2)$

Yes

Not effected

73

Proportion experiencing symptom resolution on palmoplantar areas $[N=32], n(\%)$

No

Yes

Not effected

143

Proportion experiencing symptom resolution on intertriginous areas $[N=67], n(\%)$

No

Yes

Not effected

108

Proportion experiencing symptom resolution on nails

$$
[N=26], n(\%)
$$

No

Yes

Not effected

149

BSA body surface area, $P S O$ plaque psoriasis

${ }^{a}$ Special areas include: scalp, palms, genitals, anal area, areas on your body that flex, soles of feet, and nails

previously (two to three agents) and potentially light therapy as well, who were naïve to systemic psoriasis therapy.
In addition to the symptom burden, it is important that clinicians consider the mental health burden of psoriasis; about one-third of patients reported depression/anxiety (32.0\%), which is consistent with previous research [3-5]. Furthermore, the association of psoriasis with cardiometabolic comorbidities (high cholesterol, blood pressure, and diabetes) was also observed in the patients $[22,23]$. In the current study, $<3 \%$ of patients with mild-tomoderate PSO had PsA diagnosis. The prevalence of PsA could be higher when diagnosed by rheumatologists [24] or the proportion may be lower when the condition is self-reported [25]. Another reason for low prevalence of PsA could be the study cohort of patients with mild-tomoderate severity of PSO [26-28] and the duration of the disease [29].

Most patients with mild-to-moderate PSO reported improvement in the "visible skin symptoms," "other non-skin-related symptoms," and "social/emotional well-being" as the top three treatment goals for prior and current topical therapy. These goals did not change much from the prior topical treatment to the current topical treatment, and the suboptimal benefit highlighted in our study by the patients taking topical treatments may provide insight into why assessing patients' experience and treatment satisfaction is necessary. Similar treatment goals were reported in other studies including a US cross-sectional survey [30], a qualitative study on Dutch patients with psoriasis [31], and German Psoriasis Registry [32]. Based on our findings and prior reports, treatment goals of patients with psoriasis appear to be consistent, regardless of the severity.

In our study, patients using prescription topicals with "mild" PSO severity perceived their symptoms as "moderate" in severity prior and after initiating the current treatment (scores of 7.0 and 5.0; scale of 0 "very mild" to 10 "very severe"), which is in agreement with other studies. A Nordic Patient survey of psoriasis and PsA reported that patients' perceptions of psoriasis severity correlated weakly with the severity (based on affected \% BSA) of clinical symptoms [33]. The Multinational Assessment of Psoriasis and Psoriatic Arthritis survey also reported that patients' perception of the disease 


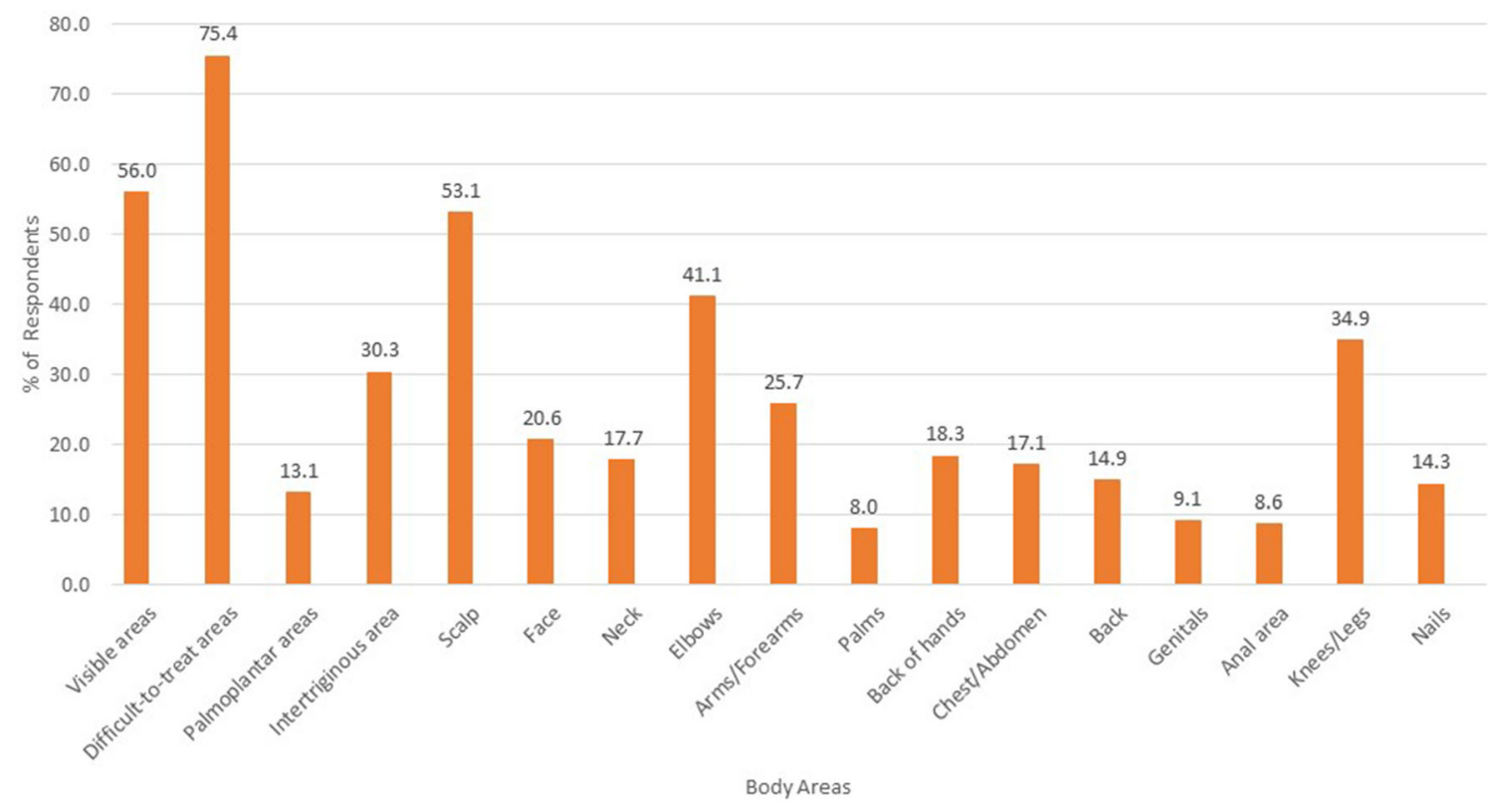

Fig. 2 Patients with mild-to-moderate PSO and on current prescription topical experiencing symptoms by body area. PSO plaque psoriasis. Visible areas: face, neck, palms, back of hands, nails. Special areas: scalp, palms,

severity may differ from their treating clinicians' perspective [34]. Itching was reported by $36 \%$ of patients as the most important factor contributing to disease severity, whereas only $12 \%$ of dermatologists felt the same. Patient's perception of the disease severity is mostly based on symptoms, including itching, location, and size of the lesions [34]. From the previously published papers, it appears that patients with psoriasis in both the US [34] and globally [35] identify itching as a measure of disease severity. However, many other factors, including the topology as well as emotional/social aspects of the disease, are known to have an impact on the QoL and affect the patient-rated severity.

In terms of visible skin symptoms, patients with the mild-to-moderate PSO had a median BSA reduction of $0 \%$ with their current topical treatment. Only $5.7 \%$ of the patients reported $\geq 75 \%$ reduction in BSA, and only $38.3 \%$ reported clear or almost clear BSA after the treatment. Approximately $43.4 \%$ of the patients showed no change in the reduction of number of palm-sized psoriasis plaques/lesions. This is genitals, anal area, areas on your body that flex, soles of feet, nails. Palmoplantar areas: palms, soles of feet. Intertriginous areas: genitals, anal area, areas on your body that flex

suggestive of inadequacy of current treatment in terms of skin clearance with prescription topicals.

Persistent PSO in one or more special body areas was reported by $93.0 \%$ of the patients, considering signs and symptoms prior to the current treatment which remained while on the current treatment. Few patients experienced resolution of PSO symptoms specifically on the nails (3.8\%), scalp (8.8\%), intertriginous $(20.9 \%)$, and palmoplantar area $(28.1 \%)$. Management of the disease in these body areas is challenging because these areas remain largely underdiagnosed and undertreated; the lack of treatment options with strong supporting evidence and the burden of application may further complicate the treatment for patients. In addition, topical treatments do not affect underlying systemic inflammatory pathways [14] and can cause irritation when used in certain areas (e.g., skin folds) [36].

Most patients $(96.0 \%)$ reported itchiness, soreness, pain, or stinging on the prior topical, whereas persistence of these symptoms was reported by $86.9 \%$ of the patients on the current 
topical medication, demonstrating that prescribed topicals were not providing satisfactory relief from these common symptoms. In addition, embarrassment/self-consciousness because of skin symptoms persisted in the majority of patients, further highlighting unmet need from a humanistic perspective.

TSQM scores indicated room for improvement (median scores ranged from 58 to 72 ) in terms of efficacy, global satisfaction, and convenience except for side effects (median score: 100). Overall, the modest treatment satisfaction may be attributed to side effects, convenience, and global satisfaction scores with topical therapy. A previous study, including patients with PSO predominantly using topical medication, reported similar TSQM-9 scores, ranging between 60 and 75 [37]. Another study reported similar TSQM-9 scores ranging between 68 and 74 for a topical suspension of calcipotriene and betamethasone dipropionate in patients with PSO [38]. These scores are comparatively lower than those reported in a previous study, where TSQM-9 scores ranged between 68 and 81 in patients with moderate PSO using betamethasone dipropionate spray for 28 days [39]. There may be variation to consider among specific topical treatments and patient populations; however, the results should also be considered in the light of the treatment duration. For example, these TSQM-9 scores were reported after a short-term treatment of 28 days from a prior study [39].

In this study, approximately half of the patients reported being non-highly adherent (highly adherent: $\geq 80 \%$ followed the treatment as instructed by the product insert) to their currently prescribed topical treatment and approximately $21.1 \%$ reported not being highly or usually adherent (usually adherent: 60-79\% followed the treatment as instructed by the product insert). Previously published global studies have also reported similar rates of high adherence, ranging between 50 and 70\% [40-43]. Challenges and reasons for nonadherence reported in the literature include cosmetic characteristics of topicals, low efficacy, fear of side effects, frequency of application, staining of clothes and bedding, familial problems, forgetfulness, and cost [42]. Although some of these may require more complex problem solving, others appear to be a direct function of the prescribed treatments themselves (e.g., forgetfulness vs. difficulty in application). In our study, it was observed that approximately half of the patients were nonadherent to prescribed topicals because of multiple reasons such as forgetfulness and other challenges associated with prescription topicals, including its frequency, consistency, and difficulty in application.

This survey highlights an unmet need in the management of mild-to-moderate PSO when using prescription topical treatments, demonstrating that successful treatment of mild-tomoderate psoriasis should consider the topology (i.e., the number and distribution of body sites affected), the specific psoriasis symptoms (e.g., itch and pain), the patient's long-term treatment goals, and the cost and burden of treatment [44]. Patients' feedback regarding treatment satisfaction and adherence behavior is critical in therapeutic success.

\section{LIMITATIONS}

This study had some limitations. This survey was conducted online at the beginning of the COVID-19 pandemic (Q1 2020); hence, there could be potential biases in terms of respondent selection and the cohort recruited, which impact generalizability. Patients included in the study, with median age of 55 years, had mild-tomoderate PSO (for $>10$ years) on prescription topicals, representing a particular segment of the PSO-afflicted patients. This online survey may not have recruited sufficiently in certain segments, such as those under institutionalized care or those not familiar with using the internet; most were commercially insured. Our study may be subject to recall bias and consequent under- or misreporting of comorbidities, PSO diagnosis timing, past psoriasis medications, psoriatic disease characteristics, and adherence. Although patients who tried more than one prescribed topical previously were included in the survey, we did not evaluate the effect of prior topical treatment on the current outcomes. We also did not compare all the 
120

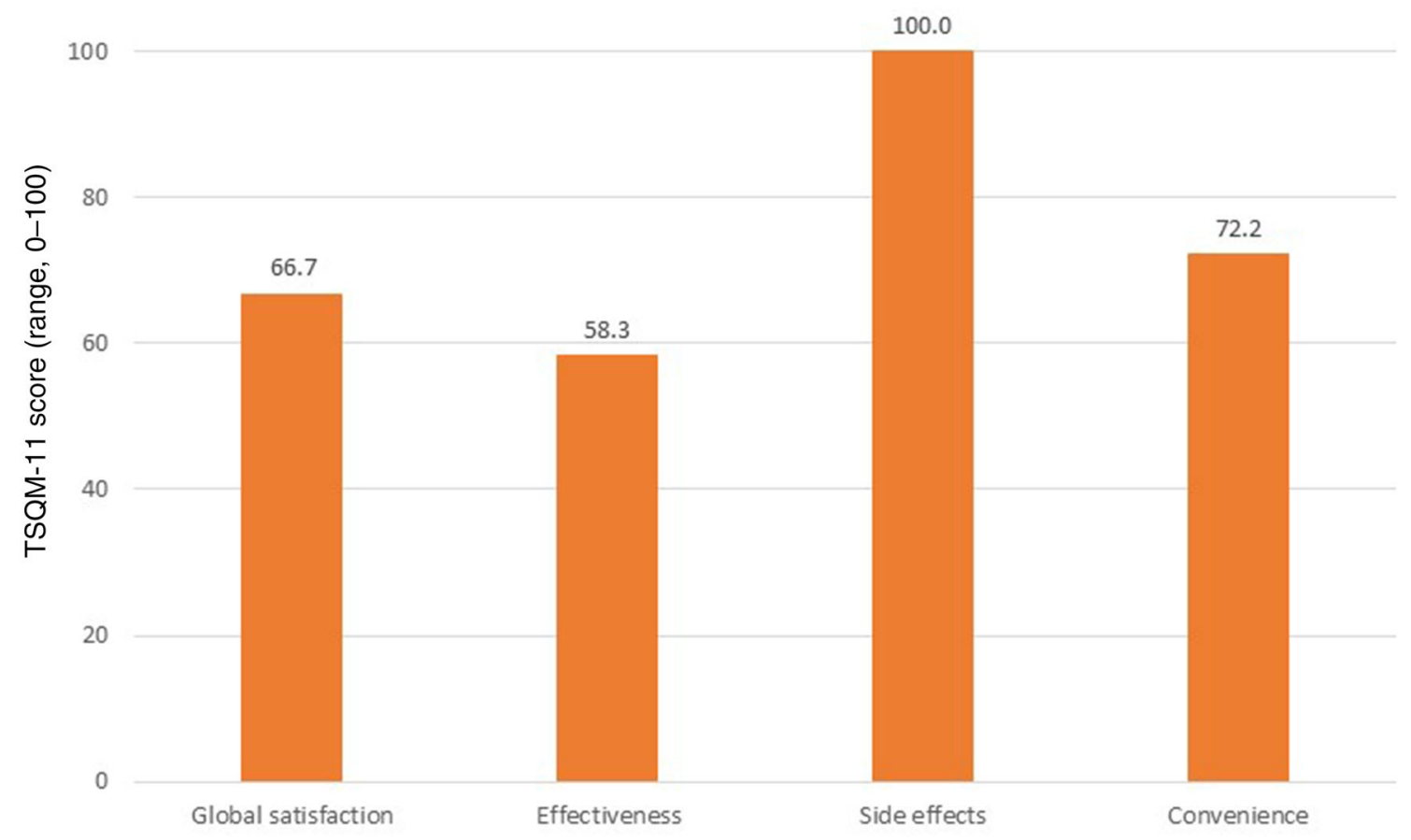

Fig. 3 TSQM-11 outcomes. TSQM treatment satisfaction questionnaire for medication

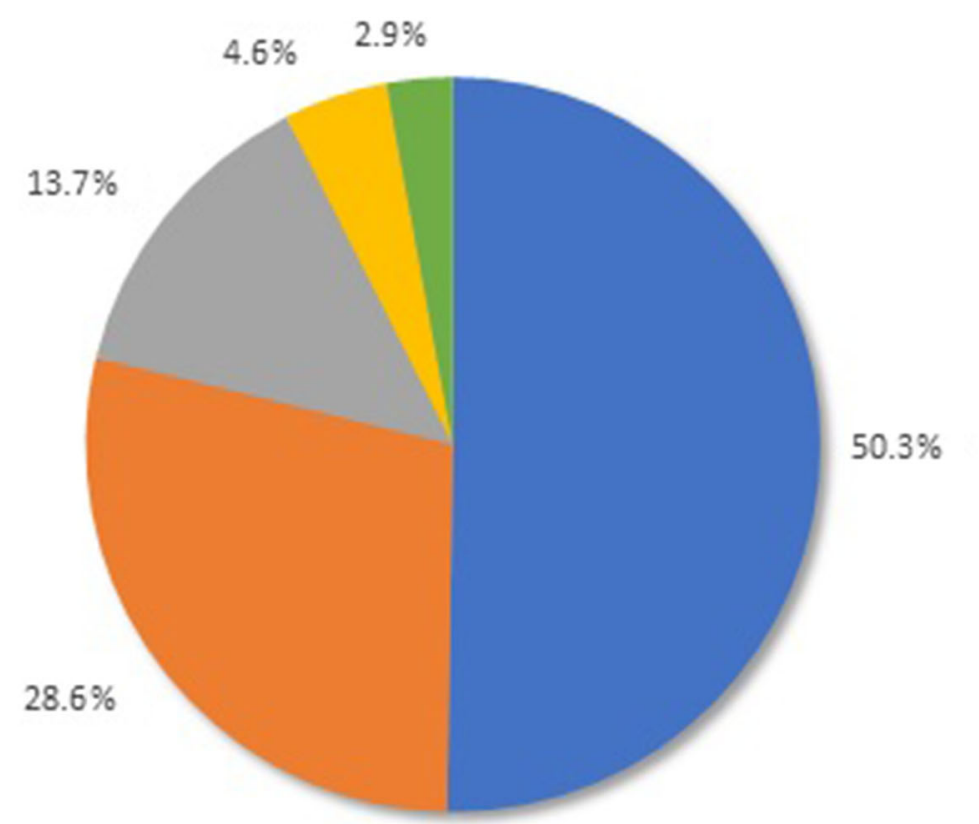

Fig. 4 Current treatment adherence in patients with mild-to-moderate PSO. PSO plaque psoriasis 


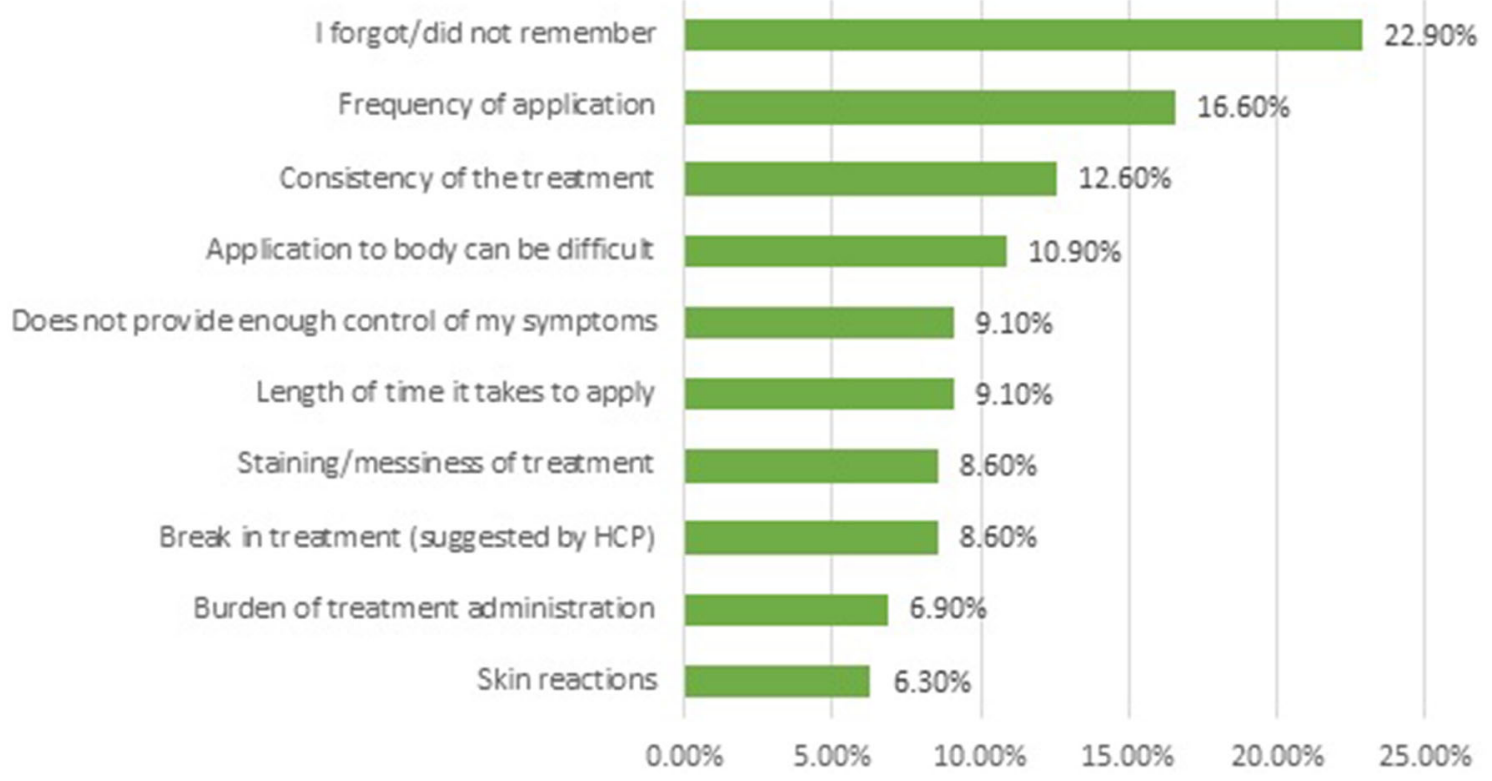

Fig. 5 Top ten reasons for non-adherence in patients with mild-to-moderate PSO. HCP healthcare provider, PSO plaque psoriasis

outcomes of before or current treatment because of burdensomeness and recall bias associated with required questions to do so nor did we collect the specific information on the specific topicals taken by patients in our survey, because of similar reasoning. Although there is a wide array of available topical treatments, each with specific attributes, we did not collect data on the number of patients using other topicals (vitamin D analogs, vitamin A derivatives, calcineurin inhibitors, tar, and steroids), which may confound meaningful conclusions regarding specific topicals. It is possible that failure or disenchantment with consecutive topicals may have led to inertia or influenced outcomes reporting. The number of patients who experienced certain symptoms and achieved symptom resolution in certain body areas reflects a convenience sample within the overall cohort we studied and would here reflect smaller subgroups with varying and potentially insufficient sample size; studies that focus on these specific subgroups within PSO should be undertaken. Additional forms of psoriasis that influenced outcomes may have been present. Finally, as we did not perform any statistical testing, no causal conclusions can be drawn from these observations.

\section{CONCLUSION}

Despite receiving three prescription topicals and being on current treatment for at least 5 years, patients with mild-to-moderate psoriasis did not achieve improvement in BSA, specific skin symptoms, and special body areas affected; reported partial treatment satisfaction; and relayed their suboptimal treatment adherence. These observations collectively demonstrate that unmet needs exist in the management of mild-to-moderate PSO when using prescribed topical treatments. Therapeutic options that can provide greater improvement in the visible skin symptoms, non-skin-related symptoms, and social and emotional well-being along with better patient satisfaction while incurring less burden to the patient and clinician are key for the management of patients with mild-tomoderate PSO.

\section{ACKNOWLEDGEMENTS}

Funding. This study was funded by Celgene. Amgen acquired the worldwide rights to 
Otezla $^{\circledR}$ (apremilast) on November 21, 2019. Amgen funded the journal's rapid service fee.

Authorship. All named authors meet the International Committee of Medical Journal Editors (ICMJE) criteria for authorship for this article, take responsibility for the integrity of the work as a whole, and have given their approval for this version to be published.

Author Contributions. All authors contributed equally in the concept, design, and execution of the study. Shaloo Gupta, Tara Nazareth, Ibrahim Khilfeh, and Halley Costantino prepared the manuscript draft. All authors reviewed the manuscript draft. Halley Costantino was responsible for the statistical analysis for the study.

Medical Writing, Editorial, and Other Assistance. The authors would like to thank Natalie Nunez-Gomez, MD (employee of Celgene at the time this study was conducted), for her contributions to study oversight, design, and execution. Medical writing support was funded by Amgen Inc. and was provided by Ashwini Atre, PhD, and Ramu Periyasamy, PhD, from Indegene Pvt. Ltd. and Cathryn M. Carter, MS, an Amgen employee and shareholder.

Disclosures. Shaloo Gupta and Halley Costantino are employees of Kantar Health and consultants to Amgen Inc. Steven Garbarini was an employee of Kantar Health at the time this study was conducted. Tara Nazareth and Ibrahim Khilfeh were employees of Amgen Inc. at the time this study was conducted. David Kaplan is an employee of Adult and Pediatric Dermatology and a consultant to Amgen Inc.

Compliance with Ethics Guidelines. The study received approval from by Pearl IRB prior to patient engagement (www.pearlirb.com) (protocol no. 18-KANT-180). All patients provided their consent electronically before completing the survey. The study was performed in accordance with the Declaration of Helsinki 1964 and its later amendments.
Data Availability. The datasets generated during and/or analyzed during the current study are available from Amgen Inc. upon request.

Open Access. This article is licensed under a Creative Commons Attribution-NonCommercial 4.0 International License, which permits any non-commercial use, sharing, adaptation, distribution and reproduction in any medium or format, as long as you give appropriate credit to the original author(s) and the source, provide a link to the Creative Commons licence, and indicate if changes were made. The images or other third party material in this article are included in the article's Creative Commons licence, unless indicated otherwise in a credit line to the material. If material is not included in the article's Creative Commons licence and your intended use is not permitted by statutory regulation or exceeds the permitted use, you will need to obtain permission directly from the copyright holder. To view a copy of this licence, visit http://creativecommons.org/licenses/by$\mathrm{nc} / 4.0 /$.

\section{REFERENCES}

1. Lebwohl M. Psoriasis. Lancet. 2003;361(9364): 1197-204.

2. Rachakonda TD, Schupp CW, Armstrong AW. Psoriasis prevalence among adults in the United States. J Am Acad Dermatol. 2014;70:512-6 (Elsevier Inc).

3. Weiss SC, Kimball AB, Liewehr DJ, Blauvelt A, Turner ML, Emanuel EJ. Quantifying the harmful effect of psoriasis on health-related quality of life. J Am Acad Dermatol. 2002;47:512-8.

4. Korman NJ, Zhao Y, Pike J, Roberts J. Relationship between psoriasis severity, clinical symptoms, quality of life and work productivity among patients in the USA. Clin Exp Dermatol. 2016;41: 514-21.

5. Strober B, Greenberg JD, Karki C, et al. Impact of psoriasis severity on patient-reported clinical symptoms, health-related quality of life and work productivity among US patients: real-world data from the Corrona Psoriasis Registry. BMJ Open. 2019;9:1-9. 
6. Armstrong AW, Schupp C, Wu J, Bebo B. Quality of life and work productivity impairment among psoriasis patients: findings from the National Psoriasis Foundation survey data 2003-2011. PLoS ONE. 2012. https://doi.org/10.1371/journal.pone. 0052935.

7. Sarkar R, Chugh S, Bansal S. General measures and quality of life issues in psoriasis. Indian Dermatol Online J. 2016;7:481.

8. Armstrong AW, Read C. Pathophysiology, clinical presentation, and treatment of psoriasis: a review. J Am Med Assoc. 2020;323:1945-60.

9. Menter A, Strober BE, Kaplan DH, et al. Joint AADNPF guidelines of care for the management and treatment of psoriasis with biologics. J Am Acad Dermatol. 2019;80:1029-72.

10. Merola JF, Qureshi A, Husni ME. Underdiagnosed and undertreated psoriasis: Nuances of treating psoriasis affecting the scalp, face, intertriginous areas, genitals, hands, feet, and nails. Dermatol Ther. 2018;31: e12589.

11. Elmets CA, Korman NJ, Prater EF, et al. Joint AADNPF Guidelines of care for the management and treatment of psoriasis with topical therapy and alternative medicine modalities for psoriasis severity measures. J Am Acad Dermatol. 2021;84:432-70 (American Academy of Dermatology, Inc.).

12. Florek AG, Wang CJ, Armstrong AW. Treatment preferences and treatment satisfaction among psoriasis patients: a systematic review. Arch Dermatol Res. 2018;310:271-319 (Springer Berlin Heidelberg).

13. Koetsier MIA, Van De Kerkhof PCM. "Patient-centered treatment" for mild-to-moderate psoriasis. J Dermatolog Treat. 2015;26:1471-753.

14. Boehncke W, Boehncke S. More than skin-deep: the many dimensions of the psoriatic disease. Swiss Med Wkly. 2014;144: w13968.

15. Samarasekera EJ, Smith CH. Psoriasis: Guidance on assessment and referral. Clin Med J R Coll Physicians Lond. 2014;14:178-82.

16. Bilal J, Berlinberg A, Bhattacharjee S, Trost J, Bin RI, Kurtzman DJB. A systematic review and metaanalysis of the efficacy and safety of the interleukin (IL)-12/23 and IL-17 inhibitors ustekinumab, secukinumab, ixekizumab, brodalumab, guselkumab and tildrakizumab for the treatment of moderate to severe plaque psoriasis. J Dermatolog Treat. 2018;29:569-78.
17. Serrano L, Maloney V, Gordon KB. Risankizumab in moderate-to-severe plaque psoriasis. Immunotherapy. 2019;11:1357-70.

18. Mui UN, Patel RR, Vangipuram R, Tyring SK. Tildrakizumab for Moderate-to-Severe Plaque Psoriasis. Skin Therapy Lett. 2019;24:1-4.

19. Rhodes J, Clay C, Phillips M. The surface area of the hand and the palm for estimating percentage of total body surface area: Results of a meta-analysis. Br J Dermatol. 2013;169:76-84.

20. Atkinson MJ, Sinha A, Hass SL, et al. Validation of a general measure of treatment satisfaction, the Treatment Satisfaction Questionnaire for Medication (TSQM), using a national panel study of chronic disease. Health Qual Life Outcomes. 2004;2:12.

21. Atkinson MJ, Kumar R, Cappelleri JC, Mass SL. Hierarchical construct validity of the Treatment Satisfaction Questionnaire for Medication (TSQM Version II) among outpatient pharmacy consumers. Value Heal. 2005;8:S9-24.

22. Edson-Heredia E, Zhu B, Lefevre C, et al. Prevalence and incidence rates of cardiovascular, autoimmune, and other diseases in patients with psoriatic or psoriatic arthritis: a retrospective study using Clinical Practice Research Datalink. J Eur Acad Dermatol Venereol. 2015;29:955-63.

23. Prodanovich S, Kirsner RS, Kravetz JD, Ma F, Martinez L, Federman DG. Association of psoriasis with coronary artery, cerebrovascular, and peripheral vascular diseases and mortality. Arch Dermatol. 2009; 145:700-3.

24. Mease PJ, Gladman DD, Papp KA, et al. Prevalence of rheumatologist-diagnosed psoriatic arthritis in patients with psoriasis in European/North American dermatology clinics. J Am Acad Dermatol. 2013;69:729-35.

25. Gelfand JM, Gladman DD, Mease PJ, et al. Epidemiology of psoriatic arthritis in the population of the United States. J Am Acad Dermatol. 2005;53: 573.e1-573.e13.

26. Alinaghi F, Calov M, Kristensen LE, et al. Prevalence of psoriatic arthritis in patients with psoriasis: a systematic review and meta-analysis of observational and clinical studies. J Am Acad Dermatol. 2019;80:251-265.e19.

27. Haroon M, Kirby B, FitzGerald O. High prevalence of psoriatic arthritis in patients with severe psoriasis with suboptimal performance of screening questionnaires. Ann Rheum Dis. 2013;72:736-40. 
28. Tey HL, Ee HL, Tan AS, Theng TS, Wong SN, Khoo SW. Risk factors associated with having psoriatic arthritis in patients with cutaneous psoriasis. J Dermatol. 2010;37:426-30.

29. Christophers E, Barker J, Griffiths C, et al. The risk of psoriatic arthritis remains constant following initial diagnosis of psoriasis among patients seen in European dermatology clinics. J Eur Acad Dermatol Venereol. 2010;24:548-54.

30. Gorelick J, Shrom D, Sikand K, et al. Understanding treatment preferences in patients with moderate to severe plaque psoriasis in the USA: results from a cross-sectional patient survey. Dermatol Ther (Heidelb). 2019;9:785-97.

31. Kouwenhoven TA, van der Ploeg JAM, van de Kerkhof PCM. Treatment goals in psoriasis from a patient perspective: a qualitative study. J Dermatol Treat. 2020;31:13-7.

32. Blome C, Gosau R, Radtke MA, et al. Patient-relevant treatment goals in psoriasis. Arch Dermatol Res. 2016;308:69-78.

33. Danielsen K, Duvetorp A, Iversen L, et al. Prevalence of psoriasis and psoriatic arthritis and patient perceptions of severity in Sweden, Norway and Denmark: results from the Nordic patient survey of psoriasis and psoriatic arthritis. Acta Derm Venereol. 2019;99:18-25.

34. Lebwohl MG, Kavanaugh A, Armstrong AW, Van Voorhees AS. US Perspectives in the management of psoriasis and psoriatic arthritis: patient and physician results from the population-based multinational assessment of psoriasis and psoriatic arthritis (MAPP) survey. Am J Clin Dermatol. 2016;17: 87-97.

35. Kerkhof PCM, Reich K, Kavanaugh A, et al. Physician perspectives in the management of psoriasis and psoriatic arthritis: results from the populationbased Multinational Assessment of Psoriasis and Psoriatic Arthritis survey. J Eur Acad Dermatol Venereol. 2015;29:2002-10.

36. Kalb RE, Bagel J, Korman NJ, et al. Treatment of intertriginous psoriasis: from the medical board of the National Psoriasis Foundation. J Am Acad Dermatol. 2009;60:120-4.

37. Ichiyama S, Ito M, Funasaka Y, et al. Assessment of medication adherence and treatment satisfaction in Japanese patients with psoriasis of various severities. J Dermatol. 2018;45:727-31.

38. Bagel J, Levi E, Tyring S, Knuckles MLF. Real-life treatment profile of calcipotriene and betamethasone dipropionate topical suspension in patients with psoriasis vulgaris. J Drugs Dermatol. 2014;13: 1374-9.

39. Fowler JF, Del Rosso JQ, Pakunlu RI, Sidgiddi S. Treatment satisfaction, product perception, and quality of life in plaque psoriasis patients using betamethasone dipropionate spray 0.05. J Clin Aesthet Dermatol. 2017;10:13-8.

40. Svendsen MT, Jeyabalan J, Andersen KE, Andersen F, Johannessen H. Worldwide utilization of topical remedies in treatment of psoriasis: a systematic review. J Dermatolog Treat. 2017;28:374-83 (Informa Healthcare USA, Inc).

41. Choi JW, Kim BR, Youn SW. Adherence to topical therapies for the treatment of psoriasis: surveys of physicians and patients. Ann Dermatol. 2017;29: 559-64.

42. Zschocke I, Mrowietz U, Karakasili E, Reich K. Nonadherence and measures to improve adherence in the topical treatment of psoriasis. J Eur Acad Dermatol Venereol. 2014;28(Suppl 2):4-9.

43. Alinia H, Moradi Tuchayi S, Smith JA, et al. Longterm adherence to topical psoriasis treatment can be abysmal: a 1-year randomized intervention study using objective electronic adherence monitoring. $\mathrm{Br}$ J Dermatol. 2017;176:759-64.

44. Segaert S, Calzavara-Pinton $\mathrm{P}$, dela Cueva $\mathrm{P}$, et al. Long-term topical management of psoriasis: the road ahead. J Dermatolog Treat. 2020;1-10. https:// doi.org/10.1080/09546634.2020.1729335. 